\title{
RETOS PARA LA ENSEÑANZA Y LA FORMACIÓN DE PROFESORES DE ESTADÍSTICA EN MÉXICO
}

\section{CHALLENGES FOR STATISTICS TEACHING AND TEACHER'S TRAINING IN MEXICO}

\author{
Sergio Hernández González* \\ Blanca Ruiz Hernández ${ }^{\dagger}$ Jesús E. Pinto Sosa \\ J. Armando Albert Huerta ${ }^{\S}$ \\ Received: 24/Feb/2012; Revised: 16/May/2013; \\ Accepted: 20/May/2013
}

\footnotetext{
* Departamento de Estadística, Universidad Veracruzana, Xalapa, México. E-Mail: sehernandez@uv.mx

${ }^{\dagger}$ Departamento de Matemáticas, Instituto Tecnológico y de Estudios Superiores de Monterrey (ITESM), Monterrey, México. E-Mail: bruiz@itesm.mx

${ }^{\ddagger}$ Facultad de Educación, Universidad Autónoma de Yucatán, Mérida, México. EMail: psosa@uady.mx

${ }^{\S}$ Misma dirección que/Same address as: B. Ruíz. E-Mail: albert@itesm.mx
} 


\title{
Resumen
}

En este trabajo se trata la problemática que se tiene en la formación y actualización docente en la enseñanza de la Probabilidad y de la Estadística en la educación superior en México. Se aborda desde cuatro enfoques: a) la caracterización y formación de profesores que impulse el desarrollo e implementación de reformas en el currículo de la enseñanza de la Estadística; b) retos del profesor en la enseñanza de la Estadística universitaria; c) las nuevas reformas curriculares y de enfoque de la enseñanza de la Estadística que proponen el desarrollo de un aprendizaje basado en proyectos con el uso de software estadístico apropiado, y d) la innovación educativa como un cuerpo de conocimiento en desarrollo, mediante el cual se promueve la conformación de redes de profesores que favorezcan el surgimiento de innovaciones reales. A partir de estas perspectivas, se plantean los retos que enfrentan la enseñanza y formación de profesores de Estadística.

Palabras clave: actualización docente, formación de profesores, enseñanza de la Estadística.

\begin{abstract}
This work will cover the problems that are found in teacher training and professional development in Probability and Statistics in higher education in Mexico. It will be approached through four focuses: a) the characterization and training of teachers that drive the development and implementation of curriculum reforms in the teaching of Statistics; b) challenges of teachers in the instruction of university-level Statistics; c) new curricular reforms with respect to the instruction of Statistics that propose the development of a learning based in projects through the use of appropriate statistical software, and d) educational innovation as a body of knowledge in development, by which the shaping of networks consisting of professors who favor the emergence of real innovation is brought about. Starting from these perspectives, the challenges confronted in the teaching and training of Statistics professors will be proposed.
\end{abstract}

Keywords: teacher professional development, teacher training, teaching of Statistics.

Mathematics Subject Classification: $97 \mathrm{C} 70$. 


\section{Introducción}

Una de las áreas plenamente reconocidas como prioritarias en el campo de la educación Estadística es la actualización y formación de profesores de Estadística. No obstante, si bien se identifican programas y cursos de formación continua para profesores (generalmente centrados en el aprendizaje de los contenidos o el manejo de algún software estadístico), la investigación en torno a la formación y caracterización de los profesores de Estadística en México es incipiente (Pinto, et al., 2007). El desarrollo de investigaciones sobre el conocimiento y la práctica del profesor de Estadística es una necesidad impostergable de atender. Así mismo, estudios como los de Pinto, et al. (2007) y Pinto (2010) dan cuenta de la diversidad de antecedentes académicos de los profesores que imparten Estadística y de la escasa o nula formación en didáctica de la disciplina en los profesores de nivel bachillerato y universitario, lo que fundamenta la importancia de contar con programas de formación inicial y de educación continua que atiendan esta necesidad.

Mientras tanto, los profesores de Estadística continuamente se enfrentan no sólo a su propia falta de preparación (consciente o inconsciente) sino a la actualización constante de una ciencia en pleno crecimiento, al surgimiento de nuevas herramientas tecnológicas y técnicas didácticas, al cambio que la sociedad impone al ámbito escolar y a las dificultades propias de la misma disciplina (Batanero, 2001). Así, mientras la investigación y los programas de actualización llegan, el profesor debe enfrentar solo su propia problemática y tratar de resolverla, aunque sea de manera parcial y provisional.

El objetivo de este trabajo es presentar la visión en torno a esta problemática que finalmente enfrentan tanto profesores como formadores de profesores de Estadística, centrando la atención en la enseñanza de la Estadística en el nivel bachillerato y universitario. Teniendo como reto la apertura a distintas perspectivas de análisis de una misma problemática que ha sido tratada con visiones diferentes desde diferentes comunidades: desde los formadores de profesores, desde la reflexión del profesor-investigador, desde la comunidad de estadísticos y desde la innovación educativa. El desarrollo de este escrito estará guiado por estas cuatro visiones. El análisis de la problemática nos llevó a reconocer los retos para la enseñanza y la formación de profesores de Estadística en México, particularmente en el nivel medio superior y superior. A mediano plazo pretendemos impulsar la formación de una comunidad de investigación en enseñanza de la Estadística en México que se enriquezca mutuamente a través de la discusión 
de diversos problemas alrededor de la enseñanza de la Estadística.

\section{Retos para la enseñanza y formación de profesores}

\section{Investigación sobre la compresión conceptual y formación del profesor}

Un primer punto de interés es sobre los procesos de aprendizaje, adquisición y desarrollo conceptual de los profesores de Estadística actuales, es decir, ¿quién es el profesor de Estadística?, ¿qué características profesionales y de formación tiene?, ¿cómo llegó a ser profesor?, ¿qué conoce?, ¿cómo surge el conocimiento del contenido y cómo evoluciona en la mente del profesor hasta ser un contenido para enseñar en la escuela?, ¿cómo lo utiliza? y ¿de qué manera, sus conocimientos y concepciones, reflejan, el tipo de formación recibida como profesor de matemáticas?

En esta línea de investigación, en México algunos estudios sobre el profesor de Estadística se han centrado en explorar sus características y necesidades de formación. Por ejemplo, Pinto, et al. (2007), en un estudio con 46 profesores de Estadística a nivel universitario, encontraron que el $78 \%$ son hombres entre 26 y 41 años de edad, el $74 \%$ labora en instituciones privadas, el $59 \%$ lo hace de tiempo parcial (por horas), cerca del $70 \%$ tiene entre 1 y 6 años de experiencia docente en Estadística, el $41 \%$ son ingenieros y $25 \%$ matemáticos. Se encontró que ningún profesor ha tomado cursos de formación y actualización en Estadística o su enseñanza. Ortega (2009), por su parte, desarrolló una investigación de carácter descriptivoexploratorio con 51 profesores de Estadística a nivel bachillerato. Halló que el $61 \%$ de los profesores se preocupan más por el logro de los objetivos de su programa de curso, caracterizando su didáctica en el uso de estrategias convencionales de enseñanza y de evaluación; uso de tareas y exámenes escritos (96\%) y el libro de texto (92\%) como recursos de apoyo.

Otros estudios (González y Pinto, 2008; Espinel, et al., 2009; Chan, 2009 y Pinto, 2010) se han centrado en explorar el conocimiento didáctico del contenido (CDC), el cual es un componente del conocimiento base para la enseñanza y busca reivindicar el valor que tiene el contenido en la didáctica de la disciplina.

Pinto (2010) investigó el CDC de dos profesores noveles (uno con formación matemática y otro psicólogo) que enseñan Estadística a estudiantes de Educación y Psicología, respectivamente. El análisis se centró en 
comprender la cognición del profesor, constituida por lo que conoce y hace y las razones por las que actúa. Los resultados dieron cuenta que los profesores sostienen una concepción diferente sobre la Estadística que sobre la representación gráfica, su aprendizaje y enseñanza; presentan algunas dificultades relacionadas con la adquisición del conocimiento tales como: relacionar el conocimiento del contenido a enseñar con las representaciones instruccionales y el conocimiento del proceso de aprendizaje del estudiante; utilizar una variedad de recursos y materiales para la enseñanza de la representación gráfica; y conocer el contenido y estudio de la representación gráfica, más allá de la construcción de gráficos.

Diferentes estudios (Bacelar, et al., 2001; Shaughnessy, 2007)) sobre el análisis de la investigación en educación estadística coinciden en la necesidad de desarrollar futuras investigaciones sobre cuestiones de enseñanza en Estadística, concepciones de los profesores sobre la Probabilidad y Estadística y su efecto en la enseñanza, el estudio sobre el conocimiento del profesor sobre el contenido y el conocimiento didáctico sobre la Estadística. Shaughnessy (2007) expresa que es necesario contestar a la siguiente pregunta ¿cuál es el conocimiento estadístico necesario para la enseñanza?

La formación de profesores de y sobre Estadística es un área con amplio reconocimiento como objeto de estudio (Pinto, 2010). Dentro de este campo, Pinto (2010) identificó una variedad de problemas objeto de preocupación de los investigadores, los cuales se pueden agrupar en los temas centrales de la investigación sobre el profesor: (a) conocimientos del profesor sobre las dificultades de aprendizaje y conocimientos, (b) la preparación y formación específica de los profesores en Estadística, (c) enseñar Estadística en contextos diferentes a la matemática, (d) los conocimientos, concepciones, creencias y actitudes del profesor, (e) el uso de las nuevas tecnologías por parte del profesor, y (f) conocimiento didáctico del contenido del profesor sobre los tópicos a enseñar.

Si bien es cierto que en los últimos años existe un progreso considerable a nivel internacional de la investigación sobre la enseñanza en el salón de clases, las cuestiones vinculadas al aprendizaje del profesor de Estadística y a su práctica son relativamente incipientes en México, particularmente en educación superior. Tanto en el campo de la educación matemática como de la Estadística, se ha demostrado que el conocimiento didáctico del contenido de los profesores juega un papel importante tanto como modelo teórico para la formación de profesores como objeto de estudio. Por ejemplo, los educadores han podido reconceptualizar el currículo, su 
interpretación e implementación en el aula escolar, así como incidir en la concepción de los programas de formación de profesores y en los procesos de enseñanza en las clases de Estadística.

\section{Principales problemas del profesor de Estadística}

El profesor de Estadística enseña una ciencia que está en continua expansión por ser parte esencial en la generación de conocimiento científico y de ingeniería y en la solución de problemas prácticos. El uso de la Estadística puede observarse en la práctica del agrónomo, por ejemplo, para medir la cantidad de hidrógeno que hay en el suelo, el biólogo para determinar el nivel de contaminación de bacterias de un cultivo, el médico para mediar la efectividad de un medicamento, el inversionista para medir el potencial de mercado o el ingeniero en sus procesos de control de calidad. Esto mismo da lugar a una gran demanda de formación en Estadística al sistema educativo, que ha delegado a la enseñanza universitaria el papel central. De ahí que el profesor tenga importantes retos de formación estadística para sí mismo y para sus jóvenes estudiantes. En los siguientes párrafos se describen algunos de ellos.

El problema de las comunidades de referencia. En una Didáctica de la Estadística actualizada, se suele recurrir a las comunidades de referencia (biólogos, agrónomos, médicos, físicos, etc.) para obtener de ellos contextos y problemas útiles para la clase que motiven a los estudiantes y provean de nuevos significados de los conceptos y técnicas Estadísticas. Sin embargo, Rodríguez, et al. (2011) muestran que esas comunidades tienen sus propios problemas para el uso adecuado de la Estadística y que deben ser tomados en cuenta para la formación universitaria. Así, por ejemplo, se encontró que una comunidad de investigación en ciencias biológicas, observada a través de sus reportes de investigación, usa el valor $p$ como determinante para decidir si su resultado era significativo, pero en su mayoría no tomaban en cuenta si el tamaño de la muestra era suficiente.

El problema de la actualización didáctica, teórica y tecnológica del profesor. La formación del profesor de Estadística no sólo se da en su fase inicial sino a través de toda su vida profesional porque su ciencia está en constante crecimiento: nuevas aplicaciones y mejoramiento de recursos tecnológicos surgen continuamente (Zapata, 2011). También la Didáctica de la Estadística está teniendo un gran desarrollo, lo cual no es nuevo, pues ya el ISI (International Statistical Institute) desde 1991 funda una sección con este propósito: la IASE (The International Association for Statistical Education). Desde entonces, la IASE a través de sus ICOTS (International 
Conference on Teaching Statistics), SERJ (Statistics Education Research Journal), IASE Round Tables, la difusión de libros y tesis doctorales, eventos, etc., ha promovido la investigación en educación Estadística y la difusión de la cultura Estadística. El reto es cómo hacer accesible de manera continuada estos saberes al profesor de Estadística.

El problema de la enseñanza-aprendizaje de la Estadística en el aula. Cualquier profesor de Estadística interesado por el aprendizaje de sus estudiantes se habrá enfrentado a dificultades de naturaleza diferente. Algunas de esas dificultades están referidas a la enseñanza misma como el qué enseñar (los contenidos más adecuados y el orden en que deben abordarse); el cómo enseñar (si a través de resolución de problemas, exposiciones, proyectos, cuándo incorporar el uso de tecnología, etc.), así como para qué enseñar (a qué necesidad de las comunidades de referencia responde la formación que está dando). Las dificultades de aprendizaje de los estudiantes suelen estar relacionadas con: cómo aprenden (sobre sus procesos de construcción de conceptos probabilísticos y estadísticos), de qué son capaces de aprender en los tiempos escolares asignados para ello, de qué es lo que realmente aprenden cuando pretendemos hacerlo de manera escolarizada, entre otras. Ante tales retos sólo hay una manera de abordarlos: colectiva, metódica y abierta. Esto es lo que pretende la investigación en Didáctica de la Estadística desarrollada a través de los últimos 20 años.

El problema de diseñar y evaluar. Al profesor de Estadística no le bastan los resultados de investigación en la enseñanza de la Estadística y sus sugerencias didácticas, sino que necesita traducirlos en el diseño de propuestas que pueda llevar al aula y darles seguimiento. Esta nueva actividad no puede ser improvisada sino que, en analogía del científico y el ingeniero que tienen en nuestra sociedad un papel claramente definido, el profesor necesita desarrollar ingeniería didáctica para afrontar los problemas que surgen en el aula para que sus estudiantes aprendan.

El problema de redes. Un profesor universitario de Estadística entiende claramente que superar las dificultades de la enseñanza de la Estadística le rebasa con mucho. Es por eso que nuestra actividad es eminentemente social, sólo en grupos colaborativos y redes es posible construir camino para superarlas. El fortalecimiento de redes colaborativas de proyectos de investigación e innovación se hacen indispensables en nuestra actividad profesional.

El problema de participar en proyectos reales. La vinculación a proyectos reales de investigación en una comunidad de referencia, si bien es re- 
comendable para otros profesores, en el caso del profesor universitario de Estadística se hace indispensable. Esta vinculación favorecería al profesor en saber qué tipo de conocimientos son más relevantes, cuáles son las dificultades más notables de la comunidad de referencia en la utilidad que hacen de la Estadística, así como tener idea de la influencia real de la formación Estadística universitaria en dicha comunidad.

\section{La necesidad de proyectos y uso de software en las clases de Estadística}

Las nuevas directrices marcadas por la Unión Europea en la Enseñanza Superior, dadas tras la declaración de Bolonia en junio de 1999 (Espacio Europeo de Educación Superior, 2006), marcaron, entre otras, la reducción del número de horas presenciales en el aula. Esto implica buscar un enfoque más práctico en muchas asignaturas instrumentales, entre las que podemos mencionar la Estadística, cuyo contenido teórico hace difícil su aprendizaje inmediato por parte de un buen número de estudiantes.

Las actuales líneas en el nuevo Espacio Europeo de Enseñanza Superior, establecen la necesidad de un cambio en el sistema actual de enseñanza en el sentido de que hay que dar más protagonismo a la capacidad y posibilidades de autoaprendizaje del alumno frente a la clásica clase magistral. En esta línea existen numerosos estudios en aras de buscar técnicas didácticas para favorecer este autoaprendizaje (ver, por ejemplo, Bricali, 2000). Una de estas técnicas es la conocida como aprendizaje basado en proyectos.

La reciente investigación en Didáctica de la Estadística ha mostrado que para proporcionar una cultura Estadística a los alumnos, no basta con enfatizar o agregar contenidos al currículo, sino que se tiene que cambiar el enfoque de su enseñanza. Una de las tendencias que surgió con mucho énfasis es la introducción de trabajo con proyectos en el salón de clase. Esta técnica didáctica permite contextualizar los conceptos y las técnicas en situaciones más acordes a la vida real y por lo tanto, propicia que cálculos como el de la media, mediana, moda, varianza, ajustar una recta de regresión a un conjunto de datos, u obtener una tabla del análisis de la varianza, adquieran un sentido dentro de la vida cotidiana o profesional del estudiante.

El uso de proyectos ha sido ampliamente documentado y puesto a disposición de los usuarios en diversas páginas Web (por ejemplo, Railsback, 2002), pero también se ha investigado y fundamentado filosóficamente. Así por ejemplo Moursund (1999/2008) describe los requisitos de esta estrate- 
gia y proporciona las bases para entender el concepto. Intel Educación (2011), por su parte, incluye una colección de planes de clase ejemplares que integran las Tecnologías de la Información y las Comunicaciones (TIC) en proyectos para el aula.

En particular en la enseñanza de la Estadística, el Grupo de Educación Estadística de la Universidad de Granada, España, se ha preocupado por documentar sus indagaciones al respecto y ponerlas a disposición del público en Batanero (2011). En esta página también se puede acceder a otras comunidades de interés, tales como la del ICOTS, ICMI, SERJ e Hipótesis Alternativa (Boletín de IASE para América Latina), así como a libros muy recomendables como los de Batanero y Godino (2001) y Batanero (2001).

Para la realización de proyectos es necesario que los alumnos usen la computadora, tanto para el análisis de datos, ya sea con un software estadístico o con una hoja de cálculo, como para elaborar sus informes en un procesador de textos. Esto significaría que los profesores requieren no sólo de tener acceso a estos paquetes sino también ser capaces de desenvolverse de manera diestra en su uso. Uno de los principales problemas es la disponibilidad. En particular el software estadístico comercial es costoso, por lo tanto, es importante pensar en la alternativa de usar software libre.

Software libre para Estadística. La disponibilidad de paquetes computacionales comerciales en el salón de clases no sólo implica que el profesor pueda tener acceso a ellos sino también que los estudiantes puedan trabajar con ellos. Algunas universidades y escuelas tratan de resolver este problema poniendo a disposición de los alumnos centros de cómputo en donde las computadoras tienen instalado el software que los profesores emplean. Pero este método, además de requerir un trámite administrativo complicado, restringe el uso del software por parte del estudiante (y en ocasiones del profesor) a las instalaciones escolares o bien al número de computadoras o paquetes que el presupuesto pueda poner al alcance de estos centros de cómputo. Esta no es una solución para nuestros actuales alumnos que, en su mayoría, tienen una computadora, que si bien generalmente no es de uso personal, al menos está en su casa. El problema se traslada, de las autoridades, al profesor, quien se ve en la necesidad de instruir y poner a disponibilidad del estudiante software estadístico que pueda obtener fácilmente y sin costo para que sus clases sean realmente interactivas y el software no se use como otra forma de exposición del profesor. Actualmente el internet provee de una gran cantidad de sitios donde obtener esta clase de software. Así por ejemplo, un sitio donde 
se puede encontrar tanto software libre, software gratuito o software de código abierto, relacionados con la Estadística, es el proporcionado por Corsini (2001-2010). Sin embargo también existe la dificultad de seleccionar y agrupar el software de mayor utilidad para el profesor de Estadística, dentro de toda la gama de opciones que ofrece el internet.

Con el fin de comenzar a atacar este problema, en una experiencia de enseñanza bajo proyectos, a los alumnos de sexto semestre de la Licenciatura en Estadística de la Universidad Veracruzana, en particular en la materia de Cómputo Estadístico II (período escolar Febrero-Agosto 2010), se les pidió investigar sobre software libre para Estadística. Como producto final se lograron explorar y escribir un pequeño manual para cada uno de los paquetes estadísticos. A continuación se presenta la descripción de solamente tres, de los 67 paquetes explorados:

- R Ver. 2.4.1. La herramienta de cómputo actualmente más utilizada en la investigación y desarrollo de la Estadística a nivel mundial. Ofrece una amplia variedad de técnicas y gráficas estadísticas como: modelación lineal y no lineal en varios estratos, pruebas estadísticas, análisis de series de tiempo, técnicas multivariantes, etc. El grupo principal de desarrollo de $\mathrm{R}$ está formado por estadísticos de primer nivel, incluyendo al autor original del lenguaje S, John Chambers de AT\&T Labs y los creadores de R, Robert Gentleman y Robert Ihaka.

- POPTOOLS Ver. 2.7. Es un complemento que se utiliza en Excel. Creado y mantenido por Greg Hood en Albano Western Australia. Maneja manipulación de matrices, simulación, Estadística univariante y multivariante, muestreo, generación de variables aleatorias y cuenta con un interesante demo de muchas técnicas estadísticas.

- Co CAEST Ver. 1.2. Elaborado en la Universidad de Valencia, España, por Juan Martínez de Lejarza. Es una potente calculadora capaz de realizar diversas funciones avanzadas relacionadas con la Estadística. Se trata de una buena herramienta pedagógica para la enseñanza de la Estadística a distintos niveles.

La reflexión final se enfoca a la utilización de software libre para el análisis de los datos, los cuales provengan de proyectos reales elaborados, tanto por los alumnos, como por el profesor, en el salón de clases. 


\section{La innovación educativa: una necesidad de nuevos marcos}

La innovación educativa es un cuerpo de conocimientos ya constituido en varias comunidades de educación en el mundo (Congreso de Investigación, Innovación y Gestión Educativas, Congreso Internacional en Innovación Educativa, Virtual Educa, entre otros) que presenta un nuevo reto para el profesor de Estadística. Las innovaciones educativas bien articuladas y el desarrollo de una cultura de innovación se han revelado como una herramienta eficaz para la transformación de las prácticas didácticas e instituciones educativas (Rivas, 2000). Sin embargo, pensar y planear un proyecto de innovación educativa no es sólo cuestión de insertar cuestiones novedosas en el salón de clases sino de hacerlo razonadamente midiendo las probabilidades de lograr su cometido de transformar algún aspecto significativo de la práctica educativa (Ortega, et al., 2007).

La innovación educativa se puede caracterizar como un cambio con mejora que implica una situación original y otra, distinta, que será el resultado de la innovación cuando en el plano personal se haya interiorizado y en el organizacional se haya institucionalizado, es decir, cuando se ha convertido en la nueva normalidad (Suárez y Ruiz, 2010, p. 262). Así el proceso innovador en el ámbito educativo es un proyecto ambicioso que suele ser lento y llevarse lustros o décadas (Ortega, et al., 2007). En este sentido, la innovación constituye la solución verificable mediante indicadores adecuados a un problema bien definido y es vista como un proceso.

Todos los cambios, mejoras e innovaciones que las reformas necesitan para transformar a la educación miran a los profesores como agentes protagónicos para lograr estos cambios y mejoras. Para desempeñar con responsabilidad este papel se requiere que los profesores adopten el modelo profesional de docencia y realicen un ejercicio sistemático de reflexión sobre su quehacer, sustentado en un marco teórico y en los aportes que las investigaciones les brindan para mejorar la calidad de los aprendizajes en sus alumnos (Ramírez, et al., 2008). Este objetivo no puede ser realizado sin el apoyo de comunidades conformadas por profesores conscientes de que su labor educativa demanda prácticas que van más allá de los esfuerzos individuales.

Así, en la enseñanza de la Probabilidad y Estadística es imprescindible el enfoque en innovaciones que propicien la reflexión del propio docente como una estrategia que favorezca no sólo el enriquecimiento paulatino de las clases de Estadística, sino también la conformación de comunidades de intercambio que apoyen la actividad individual. Acorde con esto, el obje- 
tivo de la propuesta APETIC (Actividades de Aprendizaje de Probabilidad y Estadística con uso de Tecnologías de la Información y la Comunicación) es aportar elementos que, a largo plazo, favorezcan la conformación de una comunidad de profesores e investigadores interesados en la innovación e investigación en Educación Estadística y que hagan uso de herramientas tecnológicas, particularmente editores de datos y programas de Estadística dinámica, y que tome en cuenta los estados del conocimiento de la disciplina (Ruiz, et al., 2009).

La idea del APETIC no es sólo proporcionar al profesor redes de actividades y materiales sobre los que él tenga que decidir y planear para definir secuencias que ponga en práctica en su propio curso, sino también discutir justificaciones y documentos que aporten herramientas que le faciliten la planeación de un curso de Probabilidad y Estadística y que, a su vez, proporcionen un lenguaje común para el intercambio de información entre un grupo de profesores e investigadores (Ruiz, et al., 2009). Se propone como aspecto metodológico central la idea de historiar los problemas como una actividad colectiva, que se sustenta en su caracterización según el marco de resolución de problemas (Alarcón, 1995), las evidencias del trabajo de los estudiantes y la experiencia de los profesores en problemas de estructura similar. La historia del problema es abierta y acumulativa: el trabajo sucesivo y a profundidad irá conformando historias de actividades que se robustecerán cada vez que un profesor las trabaje en clase y registre en un blog colectivo los resultados de su experiencia. Al mismo tiempo que la profundización en una actividad permitirá pasar después a la construcción de las redes de problemas y secuencias de actividades aprovechando las historias desarrolladas por el conjunto de profesores.

Desde este marco metodológico, historiar las actividades se convierte en una innovación educativa porque es una forma de recapitular y de aprovechar la experiencia docente constantemente. Las primeras versiones de la historia de una actividad pueden ser muy rudimentarias, pero rápidamente las contribuciones de los profesores la convierten en un robusto conjunto de referencias de gran utilidad. La visión que se genera es la de un profesor interesado en una mejor organización y reflexión de su propia experiencia que se enriquece con la experiencia de otros profesores que incorpora los resultados de la investigación y el uso de las TIC en su clase.

El APETIC constituye un buen ejemplo de una innovación educativa sustentada y situada dentro de una comunidad de profesores de Estadística. Sin embargo en los estudios sobre las innovaciones educativas también se ha insistido en el carácter culturalmente situado de las innova- 
ciones y en los riesgos de las transferencias ingenuas de modelos y prácticas exitosas de una región a otra (Ortega, et al., 2007). La historia de un problema, aunque flexible, se sustenta en marcos teóricos y metodológicos que deben ser compartidos por la comunidad que participa en ella y que es lo que permite la comunicación. Tener claras sus referencias internas es lo que permite una comunicación externa. Una nueva comunidad puede retomar cuestiones, siempre enmarcándose en sus propias referencias.

\section{Conclusiones}

Mientras la investigación en esta área se debate entre diferentes posturas y comunidades, los profesores, tanto de Estadística, como los formadores de profesores, tienen la tarea de resolver los problemas a los que se enfrenta diariamente en su práctica: enseñar Estadística o enseñar a enseñar Estadística. Desde las cuatro perspectivas mostradas aquí, notamos semejanzas y diferencias que podrían encaminarse a la conformación de comunidades de profesores que, respetando sus diferencias, favorezcan sus semejanzas y converjan en la generación de marcos de referencia comunes que les permitan tomar decisiones prácticas en sus aulas. La necesidad de comunidad es una las preocupaciones mencionadas por las cuatro perspectivas demarcadas y, a corto y largo plazo, la más importante.

Otra de las preocupaciones comunes es la necesidad de la enseñanza de un conocimiento en Estadística situado, que vaya más allá de la operatividad. Las reformas educativas y las tendencias de la enseñanza obligan a reflexionar sobre el contenido estadístico a enseñar, buscando centrarlo en la interpretación y significados de los objetos matemáticos. La enseñanza basada en proyectos es un recurso que permite adentrarse en este tipo de contenidos. Con este tipo de técnicas didácticas se requiere de hacer uso de datos reales o de generación de datos a través de simulaciones. El uso de software estadístico es indispensable y su adquisición puede ser facilitada por el uso de software libre que sea factible de obtener por profesores y estudiantes.

En la enseñanza universitaria, son necesarios los ejemplos en contexto, puesto que las comunidades que hacen uso de la Estadística como una herramienta para su propia investigación proporcionan reglas y métodos a la Estadística, que piden que se enseñen en las aulas universitarias. La inmersión en las exigencias de las prácticas de la comunidad a la que proporciona servicios la Estadística es necesaria para un profesor de Estadística. La investigación sobre la caracterización de la situación actual 
de los profesores se topa con la necesidad de proporcionar soluciones. Los programas de actualización permanente se mencionaron como una necesidad imperiosa. Es necesaria una acción paralela en donde se involucre al profesor como responsable de su propio aprendizaje y constructor de su práctica docente. Se requiere, no sólo actualización en la materia (en constante y continuo crecimiento) sino en conocimientos didácticos y de investigación (reflexión-indagación) del profesor que le permitan reflexionar sobre su misma práctica docente, junto con programas de acercamiento a los resultados de la investigación en la enseñanza de la Estadística. Un punto más dentro de la actualización docente es el desenvolvimiento diestro de los profesores en el uso del software estadístico y de tecnología de comunicación que puedan usar en sus clases.

El diseño de propuestas innovadoras basadas en investigación en la enseñanza de la Estadística exige un esfuerzo que se podría encausar a través de la generación de redes de comunidades de trabajo. Los programas de posgrado enfocados en la necesidad de una didáctica basada en investigación y de la innovación educativa fomentan esta generación de propuestas. Sin embargo, la innovación en el aula también se genera a través de comunidades pensantes y reflexivas de profesores interesados en propuestas innovadoras, con la ventaja de que estas estarían directamente vinculadas con la renovación que da el uso constante de esas propuestas dentro del salón de clases.

La generación de redes de profesores forzosamente se topará con la necesidad de la comunicación a distancia. Es necesaria la implementación de Portales en la Web para profesores de Estadística con el propósito de difundir investigación educativa en Estadística, materiales educativos, olimpiadas, etc., así como de fomentar el trabajo en colectivo y la generación de un lenguaje común que permita un intercambio más rico.

\section{Referencias}

[1] Alarcón, J. (1995) Notas del Seminario "Precálculo y Resolución de Problemas". DME-CINVESTAV-IPN, México.

[2] Bacelar, H.; Bright, G.; Chadjipelis, T.; Cordani, L.; Glencross, M.; Ito, P. (2001) "What are priority areas of research in statistics education?", Statistical Education Research Newsletter 2(1): 3-21.

[3] Batanero, C. (2001) Didáctica de la Estadística. Universidad de Granada, España. 
[4] Batanero, C. (2011) "Grupo de Investigación sobre didáctica de la Estadística.", in:

http://www.ugr.es/ batanero/index.html

[5] Batanero, C.; Godino, J. D.(2001) Análisis de datos y su didáctica. Materiales para la asignatura. Departamento de Didáctica de la Matemática, Universidad de Granada, España.

[6] Bricali, J. M. (2000) "Universidad 2 mil", Conferencia de Rectores de las Universidades Españolas, Madrid, España.

[7] Chan, I. (2009) Estrategias para desarrollar el conocimiento didáctico del contenido en la formación de los profesores. Monografía de Licenciatura en Educación no publicada, Universidad Autónoma de Yucatán, México.

[8] Corsini, A. (2001-2010) "Free Statistics", in:

http://freestatistics.altervista.org

[9] Espacio Europeo de Educación Superior (2006) "Declaración de Bolonia, 19 de Junio de 1999", in:

http://www. eees.es/pdf/Bolonia_ES.pdf

[10] Espinel, M.C.; González, M.T.; Bruno, A.; Pinto, J. (2009) "Las gráficas estadísticas", in: L. Serrano (Eds.) Tendencias actuales de la investigación en educación estocástica, Gráficas San Pancracio, Málaga: 133-155.

[11] González, M.; Pinto, J. (2008) "Conceptions of four pre-service teachers on graphical representations", in: C. Batanero, G. Burril, C. Reading \& A. Rossman (Eds.) Joint ICMI/IASE Study: Teaching Statistics in School Mathematics. Challenges for Teaching and Teacher Education. Proceedings of the ICMI Study 18 and 2008 IASE Round Table Conference, ICMI \& IASE, Monterrey, México.

[12] Intel Educación (2011) "Diseño de proyectos efectivos", in: http://www.intel.com/education/la/es/proyectosEfectivos/ index.html

[13] Moursund, D. (2008) "La creación de un proyecto de clase utilizando la metodología del aprendizaje por proyectos", in:

http : //www . eduteka . org/tema_mes . php3?TemaID=0007 
[14] Ortega, K. (2009) Características de la Reflexión sobre la Práctica de Profesores de Estadística en Bachillerato. Tesis de Maestría en Investigación Educativa no publicada, Universidad Autónoma de Yucatán, México.

[15] Ortega, P.; Ramírez, M.E.; Torres, J.L.; López, A.E.; Servin, C.Y; Suárez, L.; Ruiz, B. (1993) "Modelo de innovación educativa. Un marco para la formación y el desarrollo de una cultura de la innovación", Revista Iberoamericana de Educación a Distancia 10(1): $145-173$.

[16] Pinto, J. (2010) Conocimiento Didáctico del Contenido sobre la Representación de Datos Estadísticos: Estudios de Casos con Profesores de Estadística en Carreras de Psicología y Educación.. Tesis de Doctorado no publicada, Universidad de Salamanca, España

[17] Pinto, J.; Martín, G.; Barrabó, B. (2007) "Estudio de necesidades de formación de profesores que imparten Estadística en carreras del área social", in: G. Buendía Abalos \& G. Montiel Espinosa (Eds.) Memorias de la XI Escuela de Invierno en Matemática Educativa, Red de Centros de Investigación en Matemática Educativa (CIMATE), México: 451-463.

[18] Railsback, J. (2002) "Project-Based Instruction: Creating Excitement for Learning,Portland, OR: Northwest Regional Educational Laboratory", in:

http://http://educationnorthwest.org/resource/798

[19] Ramírez, M.E; Suárez, L.; Ortega, P. (2008) "Las investigaciones sobre las innovaciones educativas para la profesionalización docente en el IPN", Memorias de Virtual Educa 2008, Zaragoza, España.

[20] Rivas, M. (2000) "Innovación Educativa", Preprint, Síntesis, Madrid.

[21] Rodriguez, M.I.; Albert, J.A.; Agnelli, H.(2011) "Controversias sobre las pruebas de hipótesis: sus implicaciones para su enseñanza", Primer Encuentro Internacional de la Enseñanza en la Probabilidad y la Estadística, BUAP, Puebla, México.

[22] Ruiz, B.; Suárez, L.; Torres, J.L.; Flores, C. (2009) "Integration of technology use into statistical classroom through networks of learning activities", Proceedings of The 57th Session of the ISI International 
Statistical Institute, Durban, South Africa: ISI, South Africa. in: http://www.statssa.gov.za/isi2009/ScientificProgramme/ Ipms.aspx

[23] Shaughnessy, J.M. (2007) "Research on statistic learning and reasoning", in: F.K. Lester (Ed.) Second Handbook of Research on Mathematics Teaching and Learning, Greenwich: NCTM, Inglaterra: 9571049 .

[24] Suárez, L.; Ruiz, B. (2010) "Matemática educativa en la innovación educativa", in: R. Rodríguez, E. Aparicio, M. Jarero, S. Landy, B. Ruiz, F. Rodríguez, J. Lezama, y M. Solís. (Eds.) Memorias de la XIII Escuela de Invierno en Matemática Educativa, Red de Centros de Investigación en Matemática Educativa, México: 262-267.

[25] Zapata, L. (2011) "Algunas reflexiones acerca del conocimiento pedagógico disciplinar del profesor de Estadística", Didac 56-57, México: 9-14. 
